\title{
Geometrically constructed bases for homology of non-crossing partition lattices
}

\author{
Aisling Kenny \\ School of Mathematical Sciences \\ Dublin City University, Glasnevin, Dublin 9, Ireland \\ aisling.kenny9@mail.dcu.ie
}

Submitted: Jun 25, 2008; Accepted: Apr 10, 2009; Published: Apr 22, 2009

Mathematics Subject Classifications: 20F55

\begin{abstract}
For any finite, real reflection group $W$, we construct a geometric basis for the homology of the corresponding non-crossing partition lattice. We relate this to the basis for the homology of the corresponding intersection lattice introduced by Björner and Wachs in [4] using a general construction of a generic affine hyperplane for the central hyperplane arrangement defined by $W$.
\end{abstract}

\section{Introduction}

Let $W$ be a finite, real reflection group acting effectively on $\mathbf{R}^{n}$. In [4] Björner and Wachs construct a geometric basis for the homology of the intersection lattice associated to $W$. There is another lattice associated to $W$ called the non-crossing partition lattice. In [2], Athanasiadis, Brady and Watt prove that the non-crossing partition lattice is shellable for any finite Coxeter group $W$. Zoque constructs a basis for the top homology of the non-crossing partition lattice for the $A_{n}$ case in [11] where the basis elements are in bijection with binary trees.

A geometric model $X(c)$ of the non-crossing partition lattice is constructed in [7]. In this paper, we use $X(c)$ to construct a geometric basis for the homology of the noncrossing partition lattice that corresponds to $W$. We construct the basis by defining a homotopy equivalence between the proper part of the non-crossing partition lattice and the $(n-2)$-skeleton of $X(c)$. We exhibit an explicit embedding of the homology of the non-crossing partition lattice in the homology of the intersection lattice, using the general construction of a generic affine hyperplane $H_{\mathbf{v}}$. 


\section{Preliminaries}

We refer the reader to [5] and [8] for standard facts and notation about finite reflection groups. As in [7] we fix a fundamental chamber $C$ for the $W$-action with inward unit normals $\alpha_{1}, \ldots, \alpha_{n}$ and let $r_{1}, \ldots, r_{n}$ be the corresponding reflections. We order the inward normals so that for some $s$ with $1 \leq s \leq n$, the sets $\left\{\alpha_{1}, \ldots, \alpha_{s}\right\}$ and $\left\{\alpha_{s+1}, \ldots, \alpha_{n}\right\}$ are orthonormal. We fix a Coxeter element $c$ for $W$ where $c=r_{1} r_{2} \ldots r_{n}$. As in [7] we define a total order on roots by $\rho_{i}=r_{1} \ldots r_{i-1} \alpha_{i}$ where the $\alpha$ 's and $r$ 's are defined cyclically mod $n$. The positive roots relative to the fundamental chamber are $\left\{\rho_{1}, \rho_{2}, \ldots, \rho_{n h / 2}\right\}$ where $h$ is the order of $c$ in $W$ [10]. Let $T$ denote the reflection set of $W$. This consists of the set of reflections $r\left(\rho_{i}\right)$ where $\rho_{i}$ is a positive root and $r\left(\rho_{i}\right)$ is the reflection in the hyperplane orthogonal to $\rho_{i}$. For $w \in W$, let $\ell(w)$ denote the smallest $k$ such that $w$ can be written as a product of $k$ reflections from $T$. The partial order $\preceq$ on $W$ is defined by declaring for $u, w \in W$ :

$$
u \preceq w \Leftrightarrow \ell(w)=\ell(u)+\ell\left(u^{-1} w\right) .
$$

The subposet of elements of $W$ that weakly precede $c$ in the partial order (1) is denoted $N C P_{c}$. The subposet $N C P_{c}$ forms a lattice (by [7] for example), and is called the noncrossing partition lattice.

We now review the definition of the geometric model $X(c)$ of $N C P_{c}$ constructed in [7]. The spherical simplicial complex $X(c)$ has as vertex set the set of positive roots $\left\{\rho_{1}, \rho_{2}, \ldots, \rho_{n h / 2}\right\}$. An edge joins $\rho_{i}$ to $\rho_{j}$ if $i<j$ and $r\left(\rho_{j}\right) r\left(\rho_{i}\right)$ is a length 2 element preceding $c$. The vertices $\left\langle\rho_{i_{1}}, \ldots, \rho_{i_{k}}\right\rangle$ form a $(k-1)$-simplex if they are pairwise joined by edges. For each $w \preceq c, X(w)$ is defined to be the subcomplex of $X(c)$ consisting of those simplices whose vertices have the property that the corresponding reflections weakly precede $w$.

Finally, we recall some notation and standard facts about posets ([3], [9]). Let $P$ denote a bounded poset with minimal element $\hat{0}$ and maximal element $\hat{1}$. The proper part of the poset $P$ is denoted by $\bar{P}$ and defined to be $\bar{P}=P \backslash\{\hat{0}, \hat{1}\}$. Let $|P|$ denote the simplicial complex associated to $P$, that is the simplicial complex whose vertices are the elements of the poset $P$ and whose simplices are the non-empty finite chains in $P$. We say that the poset $P$ is contractible if the simplicial complex $|P|$ is contractible. For $\Delta$ a simplicial complex, let $P(\Delta)$ denote the poset of simplices in $\Delta$ ordered by inclusion. The barycentric subdivision of the simplicial complex $\Delta$ is the simplicial complex $|P(\Delta)|$ and is denoted $s d(\Delta)$.

\section{Homotopy Equivalence}

We begin with the observation that every simplex in $X(c)$ defines a non-crossing partition. Recall from Lemma 4.8 of [7] that if $\left\{\tau_{1}, \ldots, \tau_{k}\right\}$ is the ordered vertex set of a simplex $\sigma$ 
of $X(c)$ then

$$
\ell\left(r\left(\tau_{1}\right) \ldots r\left(\tau_{k}\right) c\right)=n-k .
$$

In particular, $r\left(\tau_{k}\right) \ldots r\left(\tau_{1}\right)$ is a non-crossing partition of length $k$.

Definition 3.1. We define $f: P(X(c)) \rightarrow N C P_{c}$ by

$$
f(\sigma)=r\left(\tau_{k}\right) \ldots r\left(\tau_{1}\right)
$$

where $\sigma$ is the simplex of $X(c)$ with ordered vertex set $\left\{\tau_{1}, \ldots, \tau_{k}\right\}$.

Lemma 3.2. The map $f$ is a poset map.

Proof. Let $\sigma=\left\{\tau_{1}, \ldots, \tau_{k}\right\} \in P(X(c))$ and let $\theta \preceq \sigma$. Therefore, $\theta=\left\{\tau_{i_{1}}, \ldots, \tau_{i_{l}}\right\}$ for some $1 \leq i_{1}<\cdots<i_{l} \leq k$. Note that for any roots $\rho$ and $\tau$, we have $r(\rho) r(\tau)=r(\tau) r\left(\rho^{\prime}\right)$, where $\rho^{\prime}=r(\tau)[\rho]$. We can use this equality to conjugate the reflections in $f(\theta)$ to the beginning of the expression for $f(\sigma)$. Therefore $f(\theta)=r\left(\tau_{i_{l}}\right) \ldots r\left(\tau_{i_{1}}\right) \preceq r\left(\tau_{k}\right) \ldots r\left(\tau_{1}\right)=$ $f(\sigma)$.

By definition of $f, f^{-1}(c)$ is the set of maximal elements in $P(X(c))$ and $f^{-1}(e)$ is empty. We therefore can consider the induced map,

$$
\hat{f}: \hat{P}(X(c)) \rightarrow \overline{N C P_{c}}
$$

where $\hat{P}(X(c))$ is the poset obtained from $P(X(c))$ by removing the maximal elements. Note that $\hat{P}(X(c))$ is the poset of simplices of the $(n-2)$-skeleton of $X(c)$.

The following result was proved by Athanasiadis and Tzanaki in Theorem 4.2 of [1] in the more general setting of generalised cluster complexes and generalised non-crossing partitions. However, we include the proof of the specific case here.

Theorem 3.3. The map $\hat{f}$ is a homotopy equivalence.

Proof. Since $f$ is a poset map by Lemma 3.2, $\hat{f}: \hat{P}(X(c)) \rightarrow \overline{N C P_{c}}$ is a poset map. We intend to apply Quillen's Fibre Lemma [9] to this map $\hat{f}$. Following the notation of [9], we define the subposet $\hat{f}_{\preceq w}$ of $\hat{P}(X(c))$ for $w \in \overline{N C P_{c}}$ by

$$
\hat{f}_{\preceq w}=\{\sigma \in \hat{P}(X(c)): \hat{f}(\sigma) \preceq w\} .
$$

We claim that $\hat{f}_{\preceq w}=P(X(w))$. Assuming the claim, the theorem follows from Proposition 1.6 of [9] if $|P(X(w))|$ is contractible. It is shown in Corollary 7.7 of [7] that $X(w)$ is contractible for all $w \in N C P_{c}$. Since $X(w)$ and $s d(X(w))$ are homeomorphic (by [9] for example) and $|P(X(w))|=s d(X(w))$, it follows that $|P(X(w))|$ is contractible.

To prove the claim we first show that $\hat{f}_{\preceq w} \subseteq P(X(w))$. If $\sigma \in \hat{f}_{\preceq w}$, then $e \prec \hat{f}(\sigma) \preceq$ $w \prec c$ by definition of $\hat{f}_{\preceq w}$. By applying Lemma 3.2 to the reflections corresponding to vertices of $\sigma$, it follows that $\sigma \in P(X(w))$. To show that $P(X(w)) \subseteq \hat{f}_{\preceq w}$, let $\sigma \in$ $P(X(w))$. If $\sigma$ has ordered vertex set $\left\{\tau_{1}, \ldots, \tau_{k}\right\}$, then $r\left(\tau_{i}\right) \preceq w$ for each $i$ by definition of $X(w)$. Then $\hat{f}(\sigma)=r\left(\tau_{k}\right) \ldots r\left(\tau_{1}\right) \preceq c$. By Equation 3.4 of [7], we know that since $\hat{f}(\sigma) \preceq c, w \preceq c$ and each $r\left(\tau_{i}\right) \preceq w$ then $\hat{f}(\sigma)=r\left(\tau_{k}\right) \ldots r\left(\tau_{1}\right) \preceq w$. Therefore, $\sigma \in \hat{f}_{\preceq w}$. 
Corollary 3.4. $\left|\overline{N C P_{c}}\right|$ has the homotopy type of a wedge of spheres, one for each facet of $X(c)$.

Proof. The map $\hat{f}$ induces a homotopy equivalence $|\hat{f}|:|\hat{P}(X(c))| \rightarrow\left|\overline{N C P_{c}}\right|$. The simplicial complex $X(c)$ is a spherical complex that is convex and contractible (Theorem 7.6 of [7]). Let $Y$ denote the subspace of $X(c)$ obtained by removing a point from the interior of each facet. Then $|\hat{P}(X(c))|$ is a deformation retract of $Y$ and therefore has the homotopy type of a wedge of $(n-2)$ spheres. The number of such spheres is equal to the number of facets of $X(c)$.

Note 3.5. This is a more direct proof of the result in Corollary 4.4 of [2] where it is proved that for a crystallographic root system, the Möbius number of $\mathrm{NCP}_{c}$ is equal to $(-1)^{n}$ times the number of maximal simplices of $X(c)$, which can also be viewed as positive clusters corresponding to the root system.

\section{Homology Embedding}

We now briefly review the results in [4] where geometric bases for the homology of intersection lattices are constructed. Let $\mathcal{A}$ be a central and essential hyperplane arrangement in $\mathbf{R}^{n}$. We refer to the connected components of $\mathbf{R}^{n} \backslash \mathcal{A}$ as regions. We let $L_{\mathcal{A}}$ denote the set of intersections of subfamilies of $\mathcal{A}$, partially ordered by reverse inclusion. We refer to $L_{\mathcal{A}}$ as the intersection lattice of $\mathcal{A}$.

Homology generators are found by using a non-zero vector $\mathbf{v}$ such that the hyperplane $H_{\mathbf{v}}$, which is through $\mathbf{v}$ and normal to $\mathbf{v}$, is generic. This means that $\operatorname{dim}\left(H_{\mathbf{v}} \cap X\right)=$ $\operatorname{dim}(X)-1$ for all $X \in L_{\mathcal{A}}$. In Theorem 4.2 of [4], it is proven that the collection of cycles $g_{R}$ corresponding to regions $R$ such that $R \cap H$ is nonempty and bounded, form a basis of $\tilde{H}_{d-2}\left(\bar{L}_{\mathcal{A}}\right)$ where $H$ is an affine hyperplane, generic with respect to $\mathcal{A}$. Lemma 4.3 of [4] states that for each region $R$, the affine slice $R \cap H_{\mathbf{v}}$ is nonempty and bounded if and only if $\mathbf{v} \cdot \mathbf{x}>0$ for all $\mathbf{x} \in R$. At this point, we refer the reader to Figure 1 which illustrates this basis for $W=C_{3}$. The figure shows the stereographic projection of the open hemisphere satisfying $\mathbf{v} \cdot \mathbf{x}>0$ and is combinatorially equivalent to the projection onto $H_{\mathbf{v}}$. Each region in the figure which is non-empty and bounded contributes a generator to the basis for the homology of the intersection lattice.

The fact that the hyperplane $H_{\mathbf{v}}$ is generic is equivalent to the fact that $0 \notin H_{\mathbf{v}}$ and $H \cap X \neq \emptyset$ for all 1-dimensional subspaces $X \in L_{\mathcal{A}}$ (Section 4 of [4]). We will refer to a non-zero vector in a one dimensional subspace $X \in L_{\mathcal{A}}$ as a ray. It is therefore sufficient to check that $H_{\mathbf{v}}$ is generic with respect to the set of rays. In Section 4.1, we describe for any $W$, the general construction of a vector $\mathbf{v}$ with $H_{\mathbf{v}}$ generic. In Section 4.2 , we use the construction of $\mathbf{v}$ to explicitly embed the homology of the non-crossing partition lattice in the homology of the intersection lattice. 


\subsection{Construction of a generic vector for general finite reflection groups}

Let $\left\{\tau_{1}, \ldots, \tau_{n}\right\}$ be an arbitrary set of linearly independent roots. Since the number of roots is finite and rays occur at the intersection of hyperplanes, it follows that the number of unit rays is finite. Hence, the set $\{\mathbf{r} \cdot \rho \mid \mathbf{r}$ a unit ray, $\rho$ a root $\}$ is finite and

$$
\lambda=\min \{|\mathbf{r} \cdot \rho|: \mathbf{r} \text { a unit ray, } \rho \text { a root and } \mathbf{r} \cdot \rho \neq 0\}
$$

is a well defined, positive, real number. It will be convenient to use the auxiliary quantity $a=1+1 / \lambda$.

Proposition 4.1. Let $\mathbf{v}=\tau_{1}+a \tau_{2}+a^{2} \tau_{3}+\cdots+a^{n-1} \tau_{n}$ and $\mathbf{r}$ be a unit length ray. Then $|\mathbf{r} \cdot \mathbf{v}| \geq \lambda$. In particular, $H_{\mathbf{v}}$ is generic.

Proof. Let $\mathbf{r}$ denote a unit length ray. Since $\left\{\tau_{1}, \ldots, \tau_{n}\right\}$ is a linearly independent set, $\mathbf{r} \cdot \tau_{k} \neq 0$ for some $\tau_{k}$. Let $k$ be the index with $1 \leq k \leq n$ satisfying

$$
\mathbf{r} \cdot \tau_{k} \neq 0, \text { and } \mathbf{r} \cdot \tau_{k+1}=0, \ldots, \mathbf{r} \cdot \tau_{n}=0
$$

By replacing $\mathbf{r}$ by $-\mathbf{r}$ if necessary, we can assume that $\mathbf{r} \cdot \tau_{k}>0$ and hence $\mathbf{r} \cdot \tau_{k} \geq \lambda$ by the definition of $\lambda$. We now compute $\mathbf{r} \cdot \mathbf{v}$.

$$
\begin{aligned}
\mathbf{r} \cdot \mathbf{v} & =\mathbf{r} \cdot\left(\tau_{1}+a \tau_{2}+a^{2} \tau_{3}+\cdots+a^{n-1} \tau_{n}\right) \\
& =\mathbf{r} \cdot \tau_{1}+a\left(\mathbf{r} \cdot \tau_{2}\right)+a^{2}\left(\mathbf{r} \cdot \tau_{3}\right)+\cdots+a^{n-1}\left(\mathbf{r} \cdot \tau_{n}\right) \\
& =\mathbf{r} \cdot \tau_{1}+a\left(\mathbf{r} \cdot \tau_{2}\right)+a^{2}\left(\mathbf{r} \cdot \tau_{3}\right)+\cdots+a^{k-1}\left(\mathbf{r} \cdot \tau_{k}\right)+0 \\
& \geq-1+a(-1)+a^{2}(-1)+\cdots+a^{k-2}(-1)+a^{k-1}(\lambda) \\
& =-1\left(1+a+a^{2}+\cdots+a^{k-2}\right)+a^{k-1}(\lambda) \\
& =\lambda .
\end{aligned}
$$

The last equality follows from the formula for the sum of a geometric series and the fact that $\lambda=1 /(a-1)$.

\subsection{Specialising the generic hyperplane}

In order to relate the homology basis for non-crossing partition lattices to the homology basis for the corresponding intersection lattice, we apply the operator $\mu=2(I-c)^{-1}$ from [7] to $X(c)$ to obtain a complex which we will call $\mu(X(c))$ and which is the positive part of the complex $\mu(A X(c))$ studied in [6]. The complex $\mu(X(c))$ has vertices $\mu\left(\rho_{1}\right), \ldots, \mu\left(\rho_{n h / 2}\right)$ and a simplex on $\mu\left(\rho_{i_{1}}\right), \ldots, \mu\left(\rho_{i_{k}}\right)$ if

$$
\rho_{1} \leq \rho_{i_{1}}<\cdots<\rho_{i_{k}} \leq \rho_{n h / 2} \text { and } \ell\left(r\left(\rho_{i_{1}}\right) \ldots r\left(\rho_{i_{k}}\right) c\right)=n-k .
$$

The walls of the facets of $\mu(A X(c))$ are hyperplanes. Since regions considered in [4] are bounded by reflection hyperplanes, this provides the connection between the two and explains why we use $\mu(X(c))$ instead of $X(c)$. 
We now apply Proposition 4.1 to the case where $\tau_{1}, \ldots, \tau_{n}$ are the last $n$ positive roots. Thus we set $\tau_{i}=\rho_{n h / 2-n+i}$. Since $\left\{\tau_{1}, \ldots, \tau_{n}\right\}$ is a set of consecutive roots and $r\left(\tau_{n}\right) \ldots r\left(\tau_{1}\right)=c$, the set $\left\{\tau_{1}, \ldots, \tau_{n}\right\}$ is linearly independent by Note 3.1 of [7].

Proposition 4.2. For $\tau_{i}=\rho_{n h / 2-n+i}$ and

$$
\mathbf{v}=\tau_{1}+a \tau_{2}+a^{2} \tau_{3}+\cdots+a^{n-1} \tau_{n},
$$

$\mu\left(\rho_{i}\right) \cdot \mathbf{v}>0$ for all $1 \leq i \leq n h / 2$.

Proof. Recall from Proposition 4.6 of [7] that the following properties hold.

$$
\begin{gathered}
\mu\left(\rho_{i}\right) \cdot \rho_{j} \geq 0 \text { for } 1 \leq i \leq j \leq n h / 2 . \\
\mu\left(\rho_{i+t}\right) \cdot \rho_{i}=0 \text { for } 1 \leq t \leq n-1 \text { and for all } i .
\end{gathered}
$$

Since $\tau_{1}, \ldots, \tau_{n}$ are the last $n$ positive roots, it follows that $\mu\left(\rho_{i}\right) \cdot \tau_{j} \geq 0$. Furthermore for each $\rho_{i}$, there is at least one $\tau_{j}$ with $\mu\left(\rho_{i}\right) \cdot \tau_{j}>0$ by linear independence of $\left\{\tau_{1}, \ldots, \tau_{n}\right\}$. Since all the coefficients of $\mathbf{v}$ are strictly positive, $\mu\left(\rho_{i}\right) \cdot \mathbf{v}>0$.

Proposition 4.3. The projection of $\mu(X(c))$ onto the affine hyperplane $H_{\mathbf{v}}$ where $\mathbf{v}$ is as in Proposition 4.2 induces an embedding of the homology of the non-crossing partition lattice into the homology of the corresponding intersection lattice.

Proof. Recall from Section 3 that homology generators for the non-crossing partition lattice are identified with the boundaries of facets of $X(c)$ and hence with facets of $\mu(X(c))$. On the other hand, we can use the generic vector $\mathbf{v}$ to identify homology generators of the intersection lattice with cycles $g_{R}$ corresponding to regions $R$ such that $R \cap H$ is nonempty and bounded. From [6], the boundary of each facet of $\mu(X(c))$ is a union of pieces of reflection hyperplanes. It follows that vertices $\mu\left(\rho_{i}\right)$ for $1 \leq i \leq n h / 2$ are rays and each facet of $\mu(X(c))$ projects to a union of affine slices of the form $R \cap H$. Furthermore, the projection of distinct $\mu(X(c))$ facets have disjoint interiors.

We denote the projection map by $p: \mu(X(c)) \rightarrow H$ and by $p_{*}$ the induced map from the homology of the non-crossing partition lattice to the homology of the intersection lattice. Then $p_{*}$ takes the homology generator $g_{F}^{\prime}$ corresponding to a facet $F$ of $\mu(X(c))$ to the sum of the intersection lattice homology generators $g_{R}$ corresponding to the affine slices $R \cap H$ contained in $p(F)$. That is $p_{*}\left(g_{F}^{\prime}\right)=\Sigma b_{R} g_{R}$ where $b_{R}=1$ if $R \cap H$ is contained in $p(F)$ and 0 otherwise.

To establish injectivity of $p_{*}$, we observe that $p_{*}\left(\Sigma a_{F} g_{F}^{\prime}\right)=\Sigma c_{R} g_{R}$ where $c_{R}=0$ if $R$ is not contained in $p(\mu(X(c)))$ and $c_{R}=a_{F}$ if $F$ is the unique facet satisfying $R \subseteq p(F)$. Thus $\Sigma a_{F} g_{F}^{\prime}$ is an element of $\operatorname{Ker}\left(p_{*}\right)$ if and only if $a_{F}=0$ for all $F$.

Example 4.4. For $W=C_{3}$ and for appropriate choices of fundamental domain and simple system, the relevant regions are shown in Figure 1 where $i$ represents $\mu\left(\rho_{i}\right)$. 


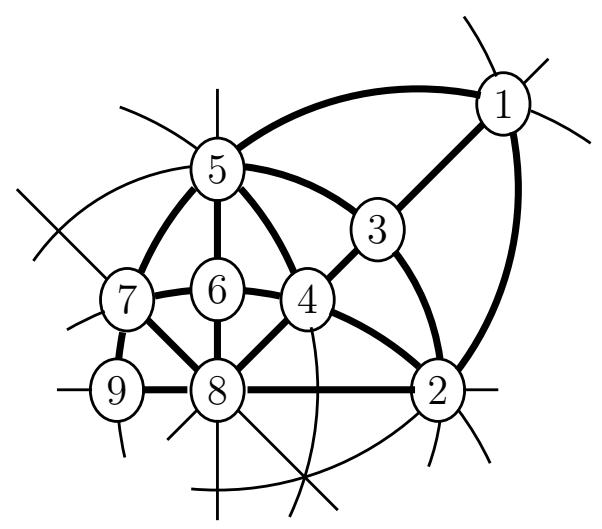

Figure 1:

The basis for homology of the intersection lattice is formed by cycles corresponding to regions in Figure 1 which are non-empty and bounded. For this example, there are 15 such regions.

Homology generators for the non-crossing partition lattice are identified with the boundaries of facets of $\mu(X(c))$, of which there are 10 in this example. These facets are outlined in bold. Note that the facet with corners $\mu\left(\rho_{2}\right), \mu\left(\rho_{4}\right), \mu\left(\rho_{8}\right)$ is a union of two facets of the Coxeter complex and therefore the embedding maps the homology element associated to this facet to the sum of the two corresponding generators in the homology of the intersection lattice.

\section{References}

[1] C.A. Athanasiadis and E. Tzanaki, Shellability and higher Cohen-Macaulay connectivity of generalized cluster complexes Israel Journal of Mathematics 167 (2008), $177-191$

[2] C.A. Athanasiadis, T. Brady and C. Watt, Shellability of noncrossing partition lattices, Proc. Amer. Math. Soc. 135 (2007), no. 4, 939-949

[3] A. Björner, Topological methods, in Handbook of combinatorics (R.L. Graham, M. Grötschel and L. Lovász, eds.), North Holland, Amsterdam, 1995, pp. 1819-1872.

[4] A. Björner and M. Wachs, Geometrically constructed bases for homology of partition lattices of type $A, B$ and D, Electron. J. Combin., 11 (2004/2006), no. 2, Research Paper 3, 26 pp. (electronic).

[5] N. Bourbaki, Lie groups and Lie algebras. Chapters 4-6. Translated from the 1968 French original by Andrew Pressley. Elements of Mathematics (Berlin). SpringerVerlag, Berlin, 2002. 
[6] T. Brady and C. Watt, From permutahedron to associahedron, arXiv:0804.2331v1 [math.CO].

[7] T. Brady and C. Watt, Noncrossing Partition Lattices in finite real reflection groups, Trans. Amer. Math. Soc. 360 (2008), 1983-2005.

[8] J.E. Humphreys, Reflection groups and Coxeter groups, Cambridge Studies in Advanced Mathematics 29, Cambridge University Press, Cambridge, England, 1990.

[9] D. Quillen, Homotopy properties of the poset of nontrivial p-subgroups of a group, Adv. in Math. 28 (1978), no. 2, 101-128.

[10] R. Steinberg, Finite reflection groups, Trans. Amer. Math. Soc. 91, No. 3, (1959) 493-504.

[11] E. Zoque, A basis for the non-crossing partition lattice top homology, J. Algebraic Combin. 23 (2006), no. 3, 231-242. 